\title{
Base de datos multicéntrica de hemorragia subaracnoidea espontánea del Grupo de Trabajo de Patología Vascular de la Sociedad Española de Neurocirugía: presentación, criterios de inclusión y desarrollo de una base de datos en internet
}

A. Lagares; P. de Toledo ${ }^{1}$; J.A. Fernández-Alén; J. Ibáñez²; F. Arikan ${ }^{3}$; R. Sarabia ${ }^{4}$; F. Ballenilla ${ }^{5}$; A. Gabarros ${ }^{6}$; A. Horcajadas $^{7}$; G. Rodríguez-Boto ${ }^{8}$; A. de la Lama ${ }^{9}$; A. Maillo ${ }^{10}$; P. Delgado ${ }^{11}$; J.L. Llacer ${ }^{12}$; J. Domínguez ${ }^{13}$; I. Arrese ${ }^{14}$

Hospital 12 de Octubre. Madrid. Grupo de Bioingeniería y Telemedicina. Universidad Politécnica de Madrid'. Hospital Son Dureta Palma de Mallorca ${ }^{2}$. Hospital Vall d'Hebron. Barcelona ${ }^{3}$. Hospital Clínico Universitario de Valladolid ${ }^{4}$. Hospital General de Alicante ${ }^{5}$. Hospital Bellvitge ${ }^{6}$. Hospital Virgen de las Nieves. Granada ${ }^{7}$. Hospital Clínico San Carlos. Madrid ${ }^{8}$. Hospital Xeral. Vigo ${ }^{9}$ Hospital Clínico Universitario. Salamanca ${ }^{10}$. Hospital General Yagüe. Burgos ${ }^{11}$. Hospital Ribera. Alcira ${ }^{12}$. Hospital Virgen de la Candelaria. Tenerife ${ }^{13}$. Hospital Donostia. San Sebastián ${ }^{14}$.

\section{Resumen}

Introducción. La hemorragia subaracnoidea (HSA) continúa siendo una de las enfermedades de interés neuroquirúrgico de más alta morbilidad y mortalidad. Su estudio es clave a la hora de mejorar la atención de estos enfermos en nuestro medio. Con este fin el Grupo de Trabajo de Patología Vascular de la SENEC decidió la creación de una base de datos multicéntrica para su estudio.

Material y métodos. Se incluyen en esta base de datos todos los casos de hemorragia subaracnoidea espontánea ingresados en los centros participantes de forma prospectiva desde Noviembre del año 2004 hasta Noviembre del 2007. Se decidieron de forma consensuada los campos a recoger incluyendo edad, antecedentes personales, características clínicas, características radiológicas y del aneurisma, tipo de tratamiento y complicaciones de la enfermedad, evolución según la escala de evolución de Glasgow (GOS) al alta y a los seis meses así como el resultado angiográfico del tratamiento. Todos los campos se recogieron en un formulario rellenable a través de una página web segura.

Resultados. En los tres años en los que ha estado activa la base se han recogido un total de 1149 casos de HSA espontánea recogidos por 14 centros participantes. Se ha estimado que es necesario aproximadamente un tiempo de 3.4 minutos para rellenar cada caso.

En cuanto a sus características generales la serie es similar a otras series hospitalarias no seleccionadas. La edad media de los enfermos incluidos es de unos $\mathbf{5 5}$ años y la relación mujer:hombre 4:3. En cuanto a la gravedad del sagrado inicial un $32 \%$ de los enfermos se encontraba en mal grado clínico (WFNS = 4 ó 5). El $5 \%$ de los pacientes fallecieron antes de realizarse una angiografía que confirmara el origen aneurismático del sangrado. Se confirmó el origen aneurismático en el $76 \%$ de los pacientes mientras que en el $19 \%$ no se encontró ninguna lesión vascular responsable del

Recibido: 26-02-08. Aceptado: 30-04-08 sangrado, siendo clasificados como HSA idiopática. En los pacientes en los que se detectó un aneurisma su tratamiento fue endovascular en el $\mathbf{4 7 \%}$ de los casos, quirúrgico en el 39 , mixto en el $3 \%$ y no recibieron tratamiento de su aneurisma el $11 \%$ de los pacientes por fallecimiento precoz. En cuanto a su evolución, la mortalidad global de la serie se sitúa en el $22 \%$. Sólo el $40 \%$ de los enfermos con HSA aneurismática presentaron una buena evolución (GOS=5).

Conclusiones. La HSA espontánea continúa siendo una enfermedad con alta morbilidad y mortalidad. Esta base de datos puede ser un instrumento para conocer mejor sus características en nuestro medio y mejorar sus resultados, ya que se trata de una serie multicéntrica hospitalaria no seleccionada. Sería pues recomendable que esta base constituyera el germen de un registro nacional de HSA espontánea.

PALABRAS CLAVE: Hemorragia subaracnoidea. Multicéntrico. Base de datos. Resultado. Aneurisma cerebral.

Spontaneous Subarachnoid Haemorrhage multicenter database from the Group for the Study of Vascular Pathology of the Spanish Society for Neurosurgery: Presentation, inclusion criteria and development of an internet-based registry

Summary

Introduction. Subarachnoid haemorrhage is one of the most severe neurosurgical diseases. Its study is crucial for improving the care of these patients in our environment. With this goal the Group for the Study of Neurovascular Pathology of the Spanish Society for Neurosurgery (SENEC) decided to create a multicenter registry for the study of this disease.

Materials and methods. In this database we have prospectively included all cases with spontaneous subarachnoid haemorrhage admitted to the participant hospitals from November 2004 to November 2007. The fields to be included in the database were selected by 
consensus, including age, past medical history, clinical characteristics at admission, radiological characteristics including presence or absence of an aneurysm and its size and location, type and complications of the aneurysm treatment, outcome assessed by the Glasgow Outcome Scale (GOS) at discharge and six months after the bleeding as well as the angiographic result of the aneurysm treatment. All fields were collected by means of an electronic form posted in secure web page.

Results. During the three years of study a total of 1149 patients have been included by 14 Hospitals. The time needed to fill in a patient in the registry is approximately 3.4 minutes. This series of patients with spontaneous SAH is similar to other non-selected in-hospital series of SAH. The mean age of the patients is $\mathbf{5 5}$ years and there is a $4: 3$ female to male ratio. In relation to the severity of the bleeding $32 \%$ of the patients were in poor clinical grade at admission (WFNS 4 or 5). $5 \%$ of the patients died before angiography could be performed. An aneurysm was confirmed as the origin of the bleeding in $76 \%$ of the patients (aSAH), while in $19 \%$ of the patients no lesion was found in the angiographic studies and were thus classified as idiopathic subarachnoid hemorrhage (ISAH). Of those patients with aSAH, $47 \%$ were treated endovascularly, $39 \%$ surgically, $3 \%$ received a combined treatment and $11 \%$ did not receive any treatment for their aneurysm because of early death. Regarding outcome, there is a $22 \%$ mortality in the series. Only $40 \%$ of the patients with aSAH reached a good outcome at discharge (GOS $=5$ ).

Conclusions. Spontaneous SAH continues to be a disease with high morbidity and mortality. This database can be an ideal instrument for improving the knowledge about this disease in our environment and to achieve better results. It would be desirable that this database could in the future be the origin of a national registry of spontaneous SAH.

KEY WORDS: Subarachnoid hemorrhage. Multicenter. Database. Outcome. Intracranial aneurysm.

\section{Introducción}

La hemorragia subaracnoidea (HSA) sigue siendo una enfermedad muy grave, que conlleva altas tasas de mortalidad y morbilidad $5,9,10,14,18,23,29,31,36$. A pesar de la introducción de nuevas técnicas de tratamiento de los aneurismas cerebrales, tales como el tratamiento endovascular, y la mejora en el tratamiento quirúrgico gracias a la difusión de las técnicas microquirúrgicas, no se ha conseguido una clara mejoría en los resultados de la enfermedad ${ }^{12,26,32}$. Parece que a pesar de la mejora en el tratamiento de la causa del sangrado, se ha avan- zado poco en cuanto al cuidado global de las complicaciones de esta enfermedad ${ }^{26,32}$.

En nuestro país no existen datos acerca de los resultados ni el tipo de tratamiento empleado en esta enfermedad más allá de los descritos en series publicadas por diferentes centros ${ }^{14,18,37}$. Existe un único registro de HSA multicéntrico elaborado en Andalucia, pero el corto periodo de recogida de casos y lo limitado del registro en cuanto al número de enfermos incluidos, hace que sea poco representativo ${ }^{21}$. A pesar de que la idea general es que el tratamiento quirúrgico de los aneurismas está siendo sustituido por el tratamiento endovascular, no existen datos sobre la frecuencia de la aplicación de cada uno de los tratamientos. Asimismo, la introducción de las técnicas endovasculares podría redundar en una mejora de los resultados finales según algunos trabajos ${ }^{19,20}$, hecho que no se ha corroborado en series globales de enfermos con $\mathrm{HSA}^{26,32}$. Por otro lado, diferentes estudios han mostrado que la frecuencia de enfermos con HSA en los que no se encuentra la causa subyacente del sangrado (HSAi) (HSA idiopática) es mayor que en otros países $8,15,18,27,28,35$. No existen datos sobre estudios multicéntricos en la literatura en la que se haya estudiado la frecuencia de HSAi.

El Grupo de Trabajo de Patología Vascular de la Sociedad Española de Neurocirugía se planteó como objetivo la elaboración de una base de datos sobre HSA. Son varios los aspectos positivos derivados de disponer de un registro multicéntrico sobre este tipo de patología. Por un lado, este registro permite disponer de información epidemiológica sobre los pacientes que sufren esta enfermedad y los factores de riesgo asociados; por otro lado, aporta información sobre factores pronósticos y su peso en la evolución final de los pacientes, facilitando a los médicos la posibilidad de informar y valorar a los pacientes de forma más adecuada. Además, permitiría conocer el tipo de tratamiento empleado, el momento en el que se aplica y en qué medida estos factores alteran el resultado final. $\mathrm{Si}$ este registro se mantuviera en el tiempo permitiría establecer la existencia de variaciones en la epidemiología y manejo de estos enfermos y de qué manera los diferentes tratamientos redundan en una mejora o detrimento del resultado final de esta enfermedad. Por todo ello, el grupo decidió elaborar de forma consensuada una base de datos sencilla y eficiente con acceso libre por parte de los diferentes servicios implicados en el estudio.

El objetivo de este trabajo es describir la elaboración de la base de datos multicéntrica del Grupo de Patología Vascular, así como su funcionamiento y los resultados globales desde su puesta en marcha en Noviembre de 2004 hasta Noviembre del año 2007. 


\section{Material y métodos}

\section{Criterios de inclusión y variables a estudio}

Se han incluido todas las hemorragias subaracnoideas espontáneas diagnosticadas en los hospitales participantes. Por tanto, se han registrado, tanto las HSAs en las que no se ha encontrado causa para el sangrado, HSAi, como aquellas en las que se ha demostrado la presencia de un aneurisma roto, HSA aneurismática confirmada (HSAa), independientemente de si se realizó algún tipo de tratamiento o no; además se incluyeron aquellos casos en los que el enfermo había fallecido antes de la realización de una arteriografía diagnóstica. El antecendente de trauma craneal o la disposición del sangrado subaracnoideo fundamentalmente en convexidad cerebral y no en cisternas basales ha sido utilizado como criterio de exclusión. El periodo de estudio comprende entre Noviembre 2004 a Noviembre 2007, si bien algunos centros comenzaron más tardíamente la inclusión de enfermos.

Las variables a estudio se determinaron de forma consensuada dentro del Grupo, intentando llegar a un mínimo número para hacer así más eficiente el sistema en cuanto a tiempo necesario para su cumplimentación por enfermo. Así se seleccionaron variables procedentes de bases de datos previamente confeccionadas por servicios integrados en el Grupo y se clasificaron en grandes grupos: identificación y antecedentes personales, forma de presentación, variables relacionadas con la angiografía, variables relacionadas con el tipo de tratamiento, complicaciones, evolución al alta y evolución final. Cada sujeto se identificó en la base mediante sus iniciales, fecha del sangrado y centro que lo incluye. Como antecedentes personales se incluyeron edad (fecha de nacimiento), sexo, antecedentes de hipertensión arterial, diabetes, tabaquismo o alcoholismo e ingesta de drogas. Se valoró la gravedad clínica del sangrado inicial mediante la escala de coma de Glasgow (GCS) ${ }^{33}$ así como la escala de la federación mundial de neurocirujanos (WFNS $)^{34}$. La cuantía del sangrado se valoró mediante la escala de Fisher ${ }^{6}$. Se registró el día en que se realizó la TC diagnóstica con respecto al día del sangrado, considerando día 1 como el día del sangrado. En cuanto a las variables relacionadas con la angiografía diagnóstica se determinó la ausencia o presencia de aneurisma responable del sangrado en la misma (HSA aneurismática o idiopática), así como el patrón de sangrado de la TC en los casos de HSA idiopática, clasificándolos en perimesencefálico (sangrado centrado anteriormente al tronco del encéfalo, con o sin extensión a la porción anterior de la cisterna ambiens y/o silviana; se admite leve teñido en cisterna interhemisférica basal y astas occipitales) ${ }^{28}$, aneurismático o TC normal en los casos en los que el diagnóstico fue hecho por punción lumbar. Se identificó la localización del aneurisma responsable del san- grado, así como la presencia de múltiples aneurismas, o la asociación con una malformación arteriovenosa. Asimismo se establecieron, bien en angiografía o bien en angio-TC el diámetro máximo del aneurisma, y la relación entre el tamaño del cuello en mm con la distancia máxima entre la base del aneurisma y la cúpula (índice cúpula/cuello). En cuanto a las variables relacionadas con el tratamiento se establecieron el tipo del mismo y el momento en el que se realizó así como el resultado angiográfico inicial. Entre las complicaciones se codificaron la ausencia o presencia de resangrado, la presencia de vasoespasmo, y la necesidad de drenaje ventricular externo o derivación de LCR definitiva. También se codificó la causa de ausencia de tratamiento del aneurisma en aquellos casos con mortalidad precoz. La evolución al alta se estableció mediante la escala de evolución de Glasgow (GOS) ${ }^{11}$ y se registró la fecha de la misma. La evolución final se estableció mediante la aplicación del GOS al menos seis meses después del sangrado. Además se registró la evolución angiográfica del aneurisma tratado, la necesidad y tipo de retratamiento y la presencia de resangrado tras el tratamiento.

\section{Soporte de la base de datos. Registro on-line en internet}

Para permitir un más fácil acceso y mejorar eficiencia del registro se decidió realizar un aplicación en Internet como soporte de la base de datos. El primer elemento a tener en cuenta en la elaboración de este tipo de soporte es la seguridad de los datos que se registran en la base. Por un lado los enfermos no son identificables en la base al ser incluidos únicamente iniciales, día del sangrado y centro como identificadores de cada caso. Por otro lado, la aplicación cuenta con otros mecanismos de seguridad como el acceso controlado con clave y nombre de usuario, distribuida únicamente a aquellos usuarios identificados en el grupo, y el uso de certificados digitales para cifrar las comunicaciones entre los ordenadores de los usuarios y el servidor. Por otro lado, se registran todos los accesos al sistema (usuario, fecha y dirección) y los intentos fallidos de acceso. La aplicación se ha escrito utilizando Application Server Pages (ASP) y javascript. Se ejecuta en un servidor web Internet Information Server y accede a los datos almacenados en una base de datos Sybase Sql Anyware v8. La disposición de las diferentes páginas que forman la aplicación así como la distribución de las variables se muestran en las Figuras 1 y 2. Se han utilizado listas desplegables y botones tipo 'checkbox' cuando las opciones son limitadas para aumentar la facilidad de uso de la aplicación. La aplicación cuenta con una página de ayuda, donde se muestran criterios de inclusión así como definiciones y ejemplos para la codificación de la información. Así mismo, se dispone de herramientas para la gestión de la base (alta de usuarios, reapertura de casos cerrados), una página de estadísticas 


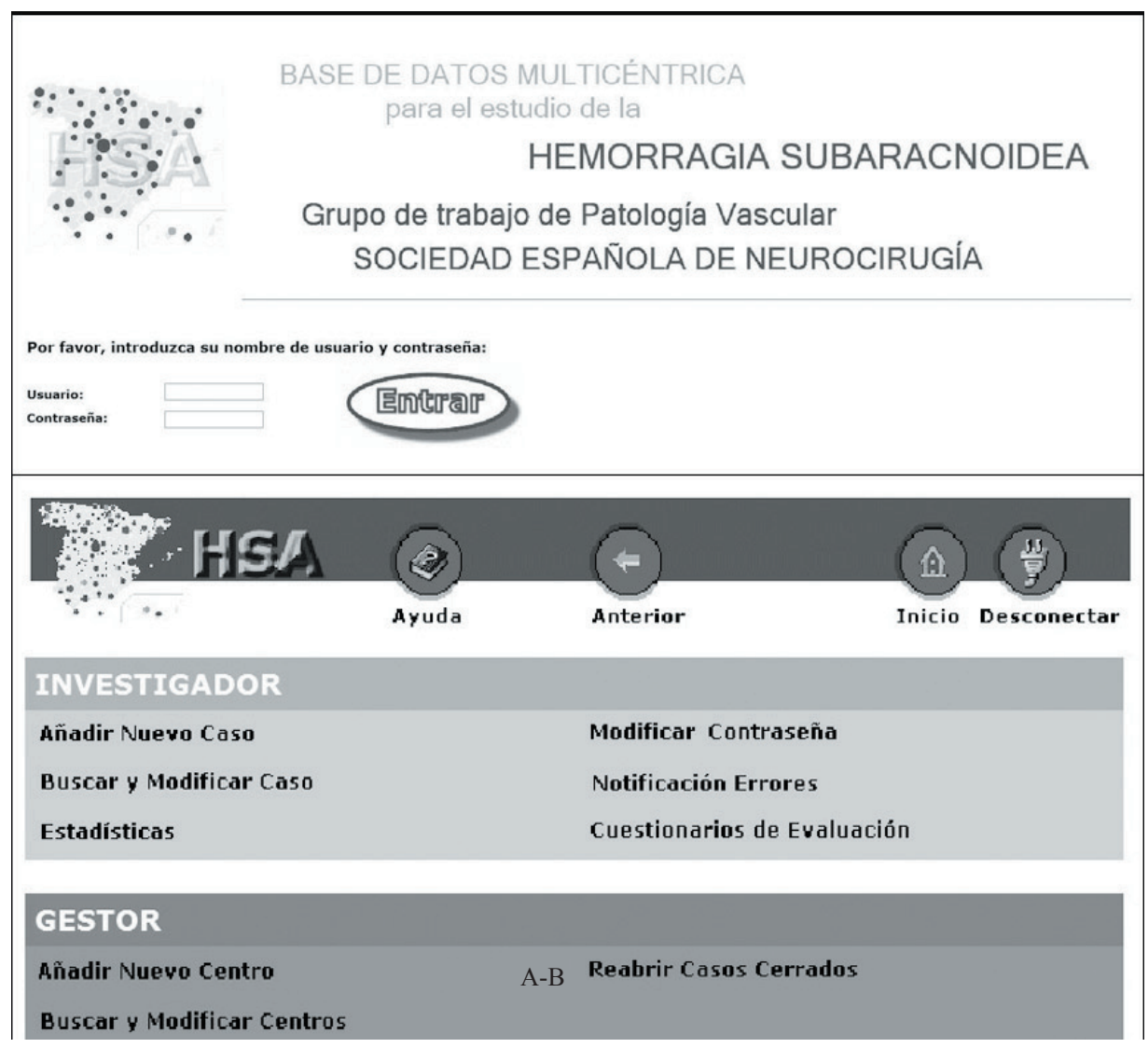

Figura 1. Página de entrada e inicial de la aplicación web, mostrando las diferentes posibilidades que presenta la aplicación.

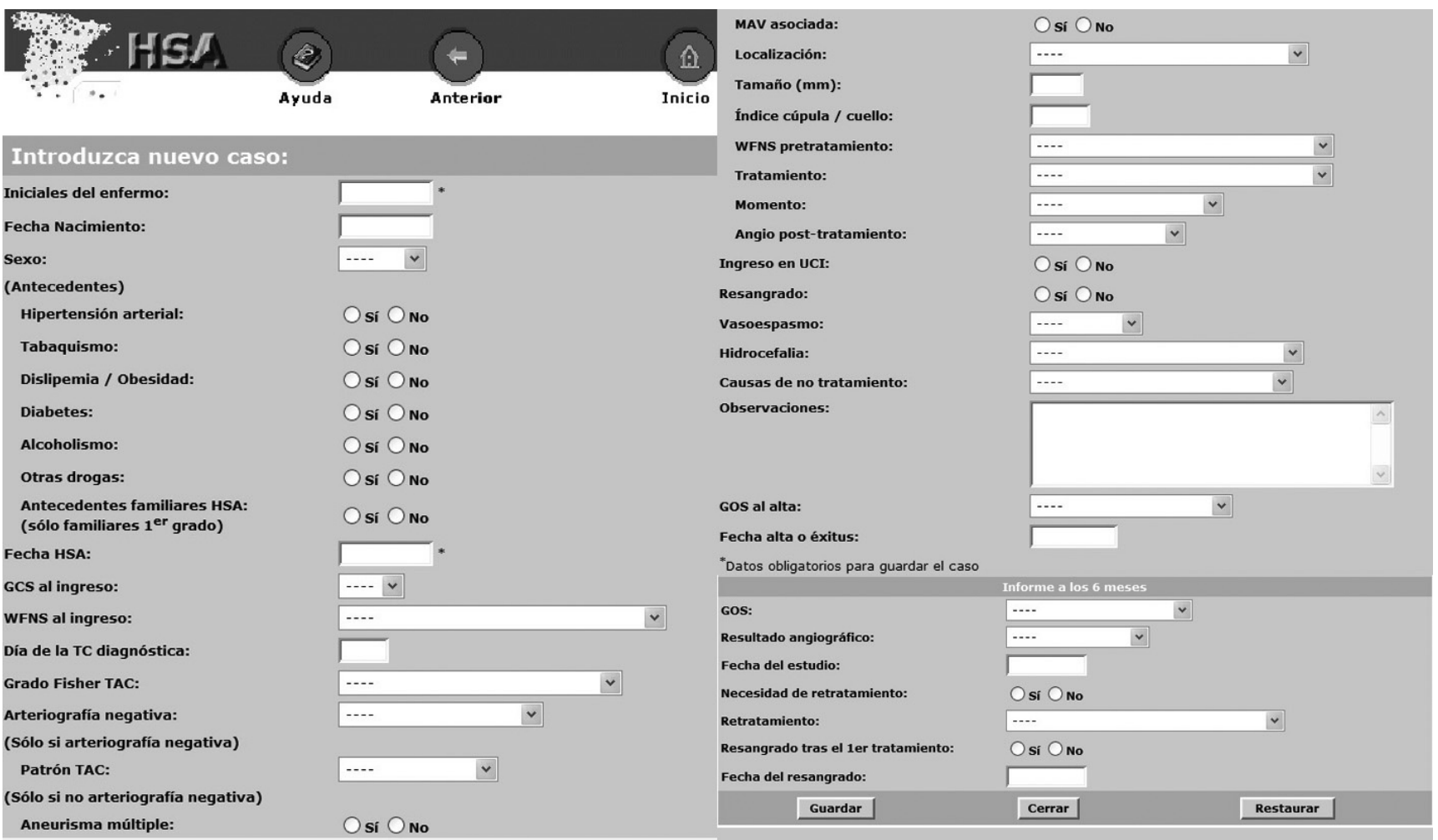

Figura 2. Hoja de recogida de datos de la aplicación web, mostrando las variables que se incluyen en el registro. 


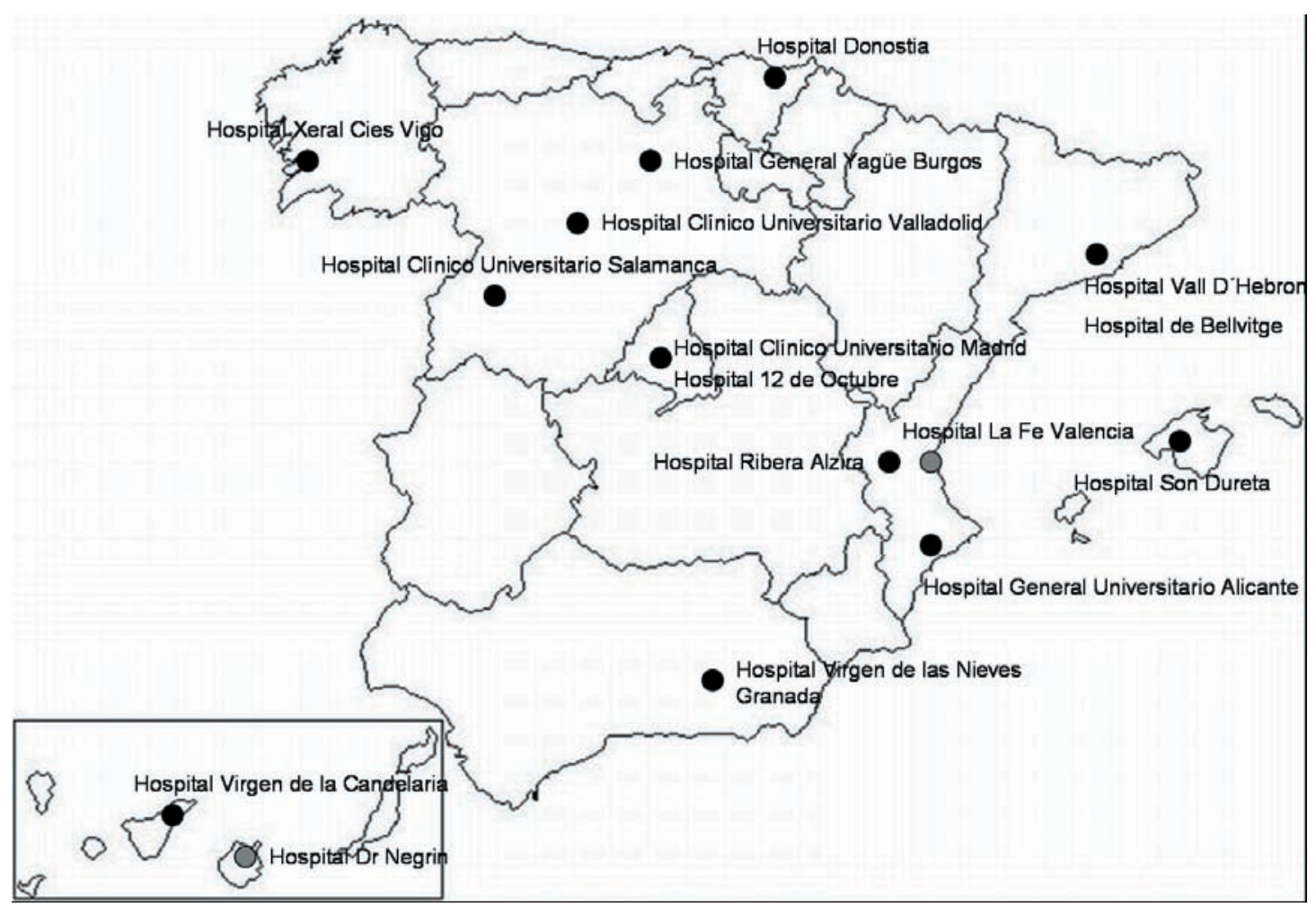

Figura 3. Distribución de los servicios que han participado en esta primera fase del estudio. En gris se muestran nuevos centros participantes.

básicas donde se recopilan brevemente algunas informaciones de la base, una zona de descarga, desde donde se puede descargar la base en formato de texto para ser usada por paquetes estadísticos tipo SPSS o EXCEL, y un cuestionario de satisfacción descargable por parte del usuario. Además, se ha implementado una opción de contacto con el administrador para la notificación de errores.

\section{Análisis de la eficiencia del registro y primeros resultados}

Para el análisis de la eficiencia de la base de datos se han utilizado el conjunto de datos derivados del registro de entradas que automáticamente realiza la aplicación. Dichas entradas pueden realizarse por diferentes motivos utilizando para el análisis sólo aquellas en las que se produjeran modificaciones en los datos de la base, para así establecer el tiempo requerido para la cumplimentación de un caso y el momento del día y semana en la que se realizaron las entradas en la base, realizándose un análisis descriptivo de estos datos.

Para el análisis de los primeros resultados se ha volcado la información presente en la base de datos desde Noviembre de 2004 hasta Noviembre de 2007 en el paquete estadístico SPSS. Se han llevado a cabo comprobaciones sobre la veracidad, consistencia y grado de cumplimentación de los datos, realizando tablas de contingencia entre variables mutuamente excluyentes. Se ha efectuado un análisis descriptivo de los datos más importantes del registro.

\section{Resultados}

En el momento de realizar este estudio se habían incluido 1149 pacientes procedentes de 14 centros, si bien durante la elaboración del mismo el número de centros se ha incrementado hasta alcanzar un total de 16 centros. Su localización queda reflejada en la Figura 3. El número aproximado de enfermos de HSA incluidos por centro al año se sitúa en torno a los 29 , si tenemos en cuenta aquellos centros que han incluido casos desde el inicio de la base.

\section{Análisis de la eficiencia del registro}

Del análisis de los accesos a la base se desprende que el formulario inicial se rellena en un tiempo medio de 3.4 minutos, siendo el tiempo para accesos con cumplimentación de casos entre el minuto y los 10 minutos en más del $90 \%$ de los casos. En cuanto al número de accesos por día de la semana, existe una distribución bastante homogénea entre los siete días, siendo el día con mayor número de entradas el lunes y el menor el sábado. 


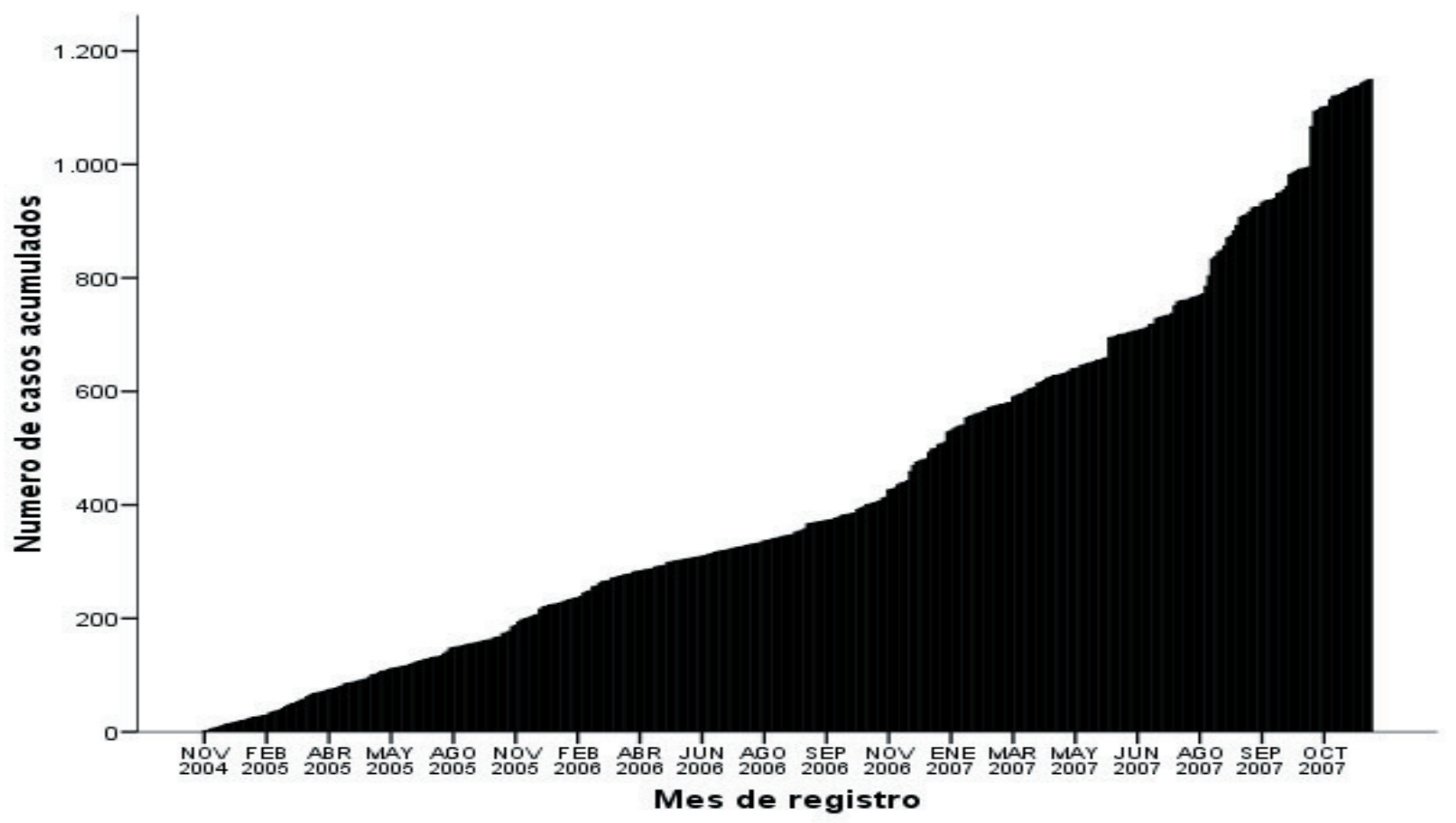

Figura 4. Ritmo mensual de inclusión de enfermos en el estudio.

En cuanto a la distribución dentro del día destaca que más de la mitad de los accesos al sistema se producen entre las 16 y las 24 horas.

No han existido incidencias relacionadas con la seguridad de los registros, ni tampoco pérdida de información.

En la Figura 4 se muestra el ritmo de reclutamiento en la base. Se observa un incremento paulatino en el número de casos incluidos, que experimenta un importante crecimiento en los últimos meses.

Características generales de los pacientes incluidos en el registro

Las características demográficas y clínicas de los pacientes incluidos en el registro se muestran en la Tabla 1. En cuanto a la distribución por edades y sexos se observa cómo esta enfermedad es más frecuente por encima de los 50 años. Es más frecuente en mujeres en todos los grupos de edad, llegando a doblarse el número de mujeres por encima de los 70 años. No se observó ningún patrón de agregación temporal, ya sea una mayor incidencia de HSA espontánea, HSAa o HSAi por meses, estaciones, o por días de la semana. En cuanto a la gravedad inicial del sangrado, aproximadamente dos tercios de los enfermos fueron ingresados en los centros participantes con un buen grado clínico. En la mayor parte de los casos los enfermos tenían un sangrado clasificable como Fisher 3 o 4 en la TC inicial.
Tabla 1

Características demográficas y clínicas de 1149 pacientes incluidos en el registro multicéntrico de HSA

Edad, media (años)

$\%$ Por grupos de edad

$<50$ años 34

50-70 años

$>70$ años

Sexo (\%)

Hombres

Mujeres

Ratio Mujer/Hombre por grupo de edad

$<50$ años

50-70 años

1,27

$>70$ años

2,06

Buen grado clínico $\quad 68 \%$

Mal grado clínico $\quad 32 \%$

Fisher 3 ó 4 


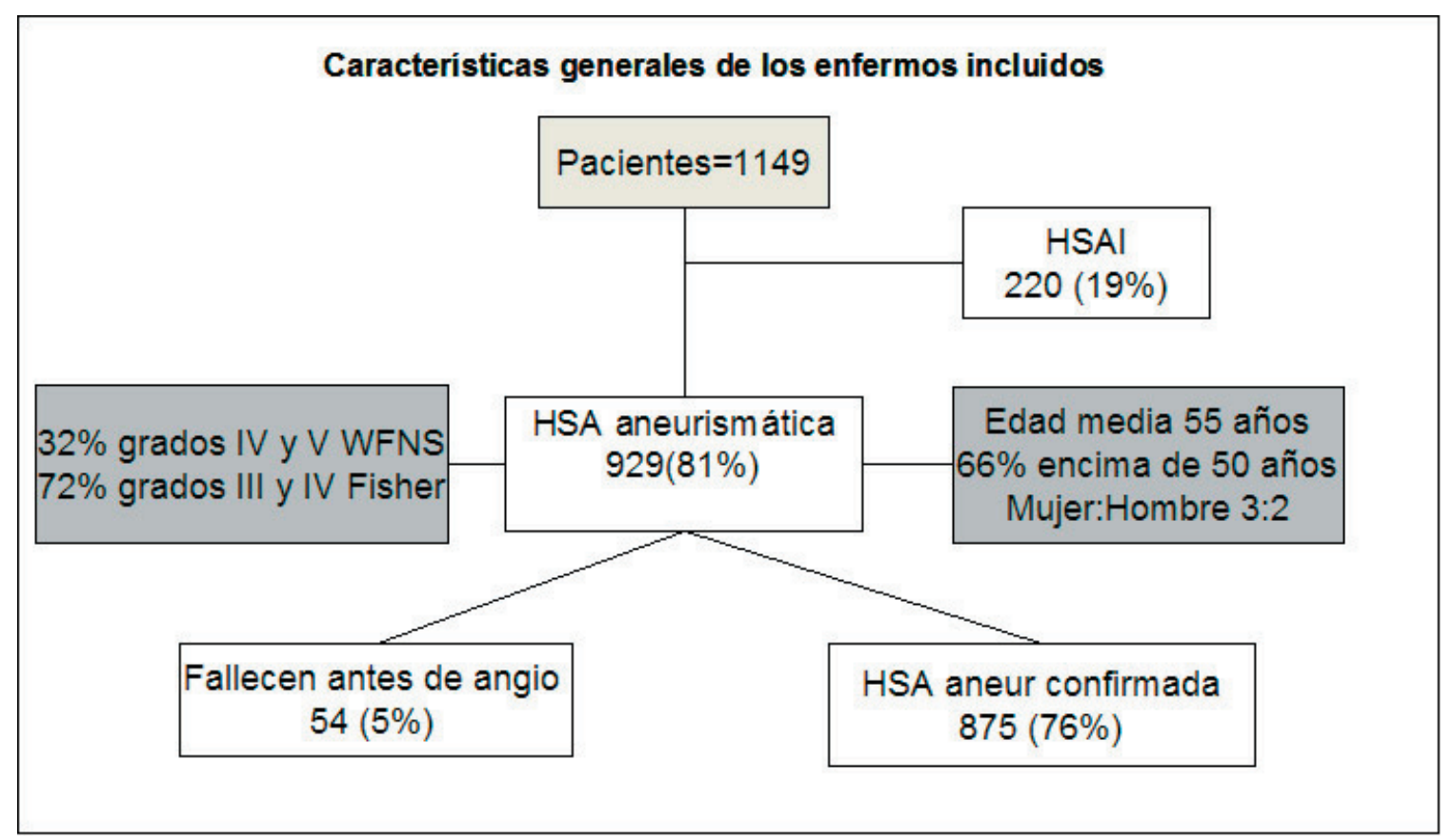

Figura 5. Características generales de los enfermos incluidos en la base.

La mayor parte de los enfermos fueron estudiados mediante angiografía, falleciendo un $5 \%$ del total de pacientes de la serie antes de poder realizar alguna prueba diagnóstica que pudiera mostrar el origen del sangrado (angiografía o TC helicoidal) (Figura 5). Del número total de pacientes, un $19 \%$ fueron clasificados como HSAi, es decir como enfermos en los que una primera angiografía resultó negativa. El resto de pacientes $(929,81 \%)$ fueron clasificados como casos HSAa, siendo confirmado su origen aneurismático en 875 , un $76 \%$ del total de la muestra. En estos pacientes el aneurisma responsable del sangrado se localizó más frecuentemente en la arteria comunicante anterior, seguido de la cerebral media y la comunicante posterior (Tabla 2). Un 19\% de los enfermos en los que se encontró un aneurisma presentaban múltiples aneurismas, presentando sólo un $1,4 \%$ una malformación arteriovenosa asociada.

El tratamiento más frecuentemente empleado en los enfermos con HSAa fue el endovascular (44\% de estos enfermos) (Tabla 3). Destaca la alta frecuencia de enfermos que aún siendo diagnosticados de un aneurisma mediante angiografía no recibieron tratamiento (11\%). En cuanto al momento de aplicación del tratamiento cabe destacar que fue realizado de forma aguda o precoz (hasta el tercer día de evolución) en casi dos tercios de la muestra.

La Tabla 4 muestra los resultados al alta para la serie global, enfermos con HSAa y enfermos que han recibido tratamiento de su aneurisma, además de la estancia media en estos casos. Cabe destacar que la mortalidad global hospitalaria asciende al $22 \%$, y que un buen resultado $(\mathrm{GOS}=5)$
Tabla 2

Características angiográficas de la serie

\begin{tabular}{lcc}
\hline Clasificación Angiográfica & Casos & $(\%)$ \\
HSA idiopática & 220 & $(19 \%)$ \\
HSA aneurismática & 929 & $(81 \%)$ \\
Localización aneurisma & $(\%)$ & \\
Comunicante anterior & 36 & \\
Cerebral media & 21 & \\
Comunicante posterior & 18 & \\
Carótida (coroidea, bifurcación) & 9,9 & 3,4 \\
Vertebral (ACPI) & 2,6 \\
Pericallosa & 2,5 \\
Basilar & 2,2 \\
Carótido-oftálmico & 2,2 & \\
ACAI, ACS & 1,4 & \\
Cerebral anterior & 0,8 \\
Cerebral posterior & 19,4 \\
Aneurismas múltiples & 1,4 & \\
Asociación a MAV & \multicolumn{2}{c}{} \\
\hline
\end{tabular}

ACPI: Arteria cerebelosa posteroinferior, ACAI: arteria cerebelosa anteroinferior, ACS: arteria cerebelosa superior.

se consiguió en menos de la mitad de los enfermos tanto en la serie global, como en el conjunto de enfermos que llegan a ser tratados. 
Tabla 3

Tipo de tratamiento y momento de aplicación en HSAa confirmada

\begin{tabular}{lc} 
Tipo de tratamiento & $(\%)$ \\
Endovascular & 47 \\
Quirurgico & 39 \\
Mixto & 3 \\
No tratamiento & 11 \\
& \\
Momento del tratamiento & $(\%)$ \\
Agudo $(<24 \mathrm{~h})$ & 28 \\
Precoz $(24 \mathrm{~h}-3$ días $)$ & 36 \\
Tardío $(>3$ días $)$ & 36 \\
\hline
\end{tabular}

\section{Discusión}

La existencia de registros nacionales de enfermedades, tan frecuentes en otras patologías, no lo son tanto en el ámbito neuroquirúrgico. Quizás desde el punto de vista neuroquirúrgico los pioneros en registros similares proceden del área de la Neurotraumatología, y en especial a través de la formación de organismos supranacionales de estudio, como es el European Brain Injury Consortium (EBIC). Existen registros similares realizados en otros países de nuestro entorno, aunque no en el área mediterránea, como es el caso de estudios poblacionales sobre la incidencia de HSA o aneurismas en Finlandia ${ }^{7}$, Suecia $^{22}$ o en algunas áreas de Gran Bretaña ${ }^{30,38}$.

Con la generalización del acceso a Internet, los portales basados en web se han convertido en la plataforma ideal para realizar proyectos de investigación que llevan aparejada la recolección de volúmenes importantes de datos. En el caso de los registros multicéntricos las ventajas son todavía más evidentes, de modo que en los últimos años se han publicado diferentes experiencias en este sentido ${ }^{25}$. Diferentes investigadores coinciden en que el principal problema de este tipo de sistemas es garantizar la seguridad de la información almacenada, especialmente si se trata de información de carácter personal ${ }^{1}$. En España los archivos con este tipo de información están sujetos a una serie de requisitos, de acuerdo con la Ley Orgánica de Proteccion de Datos de Carácter Personal (LOPD), entre los que se puede citar la necesidad de mantener un registro de todas las acciones que supongan una visualización o modificación de los datos. El registro aquí presentado no contiene datos que permitan identificar a los pacientes, y no está por tanto sujeto a dichas normas.

Las principales ventajas de este tipo de registros frente al registro en papel son obvias: reducción de errores al eliminar la necesidad de transcribir los datos, posibilidad de realizar un control de errores en los datos cuando son introducidos, y disponibilidad inmediata de la información para todos los participantes en el registro. Si los comparamos con aplicaciones informáticas dedicadas las ventajas son, por un lado, que se elimina la necesidad de tener instalado en el ordenador de cada uno de los usuarios un programa específico, lo que resulta caro y complicado de mantener, y se sustituye por un navegador genérico, y por otro, que no es necesario refundir los registros realizados por cada centro para crear el registro común ${ }^{3,16,25}$.

Los primeros registros de este tipo datan de los últimos años 90 , y hoy en día su uso es bastante generalizado. Uno de los más extensos en número de pacientes es el estudio Invest (International Verapamil SR/Trandolapril Study) ${ }^{17}$ en el área de cardiología, que acumuló 22.576 pacientes y en el que participaron 862 centros de 14 países. De las distintas fases de un estudio clínico (protocolo, registro, randomización y recolección de datos), es ésta última la que se realiza con más frecuencia usando Internet ${ }^{25}$.

$\mathrm{Si}$ comparamos esta serie con otras de la literatura internacional podemos comprobar cómo la situación en nuestro país es muy similar a la de otros países desarrollados, tanto en lo que se refiere al tipo y situación de los enfermos, como en el resultado final, siempre que los mismos se enfoquen al estudio de todos los casos y no de una serie seleccionada. Así, registros comparables al presentado en este trabajo serían series recogidas recientemente en los

\section{Tabla 4}

Resultado al alta y estancia media

HSAa tratada

Evolución (\%)

Mortalidad

Discapacidad (EV, SD, MD)

Buena recuperación
22

28

50
26

34

40
15

39

46

Estancia mediana días (RIQ)

$18(10-33)$

$20(11-36)$

23 (14-39) 
Estados Unidos mediante el estudio de los diagnósticos al alta de los enfermos publicados en los periodos 1996 a $2001^{26}$ y el recogido durante el año $2003^{32}$, o los resultados de una serie de pacientes tratados por HSA en diferentes hospitales de 18 estados realizada por Cross y colaboradores $^{4}$. Estos estudios muestran unos porcentajes de mortalidad intrahospitalaria muy parecidos a la registrada en esta serie, es decir, en torno al $25 \%$, siendo la mortalidad de las hemorragias tratadas con técnica microquirúrgica o endovascular también similar, en torno al $12-17 \%^{32}$. Estos datos son similares a los encontrados en series del Norte de Europa, con un $45 \%$ de mortalidad global en Finlandia ${ }^{7}$ (estudio poblacional en el que se conjugan mortalidad pre e intrahospitalaria), y $21 \%$ en Suecia ${ }^{31}$. Un dato a tener en cuenta en las series de manejo global hospitalario americanas es la baja frecuencia de tratamiento del aneurisma responsable del sangrado observada, que está en torno al 30$35 \%$ en diferentes series ${ }^{2,4,26,32}$, manteniéndose esta tendencia en el tiempo ${ }^{32}$. Aunque estos estudios incluyen aquellos enfermos con HSAi, el porcentaje de enfermos tratados en nuestro país es muy superior, siendo del $68 \%$ en el global de la serie y del $84 \%$ si excluimos las HSAi, aún superior al $60 \%$ esperado en algunas series americanas en las que suprimen todos aquellos enfermos que fallecen durante su ingreso hospitalario y enfermos con $\mathrm{HSAi}^{32}$. Este porcentaje es similar pero superior al registrado en series hospitalarias de manejo global de enfermos con HSA espontánea en países de nuestro entorno, como es el caso del registro de HSA de Newcastle, en el que en un periodo de 10 años el 38\% de las HSA no recibieron tratamiento alguno del aneurisma responsable del sangrado ${ }^{24}$. Cabe pensar que aunque en los estudios americanos la existencia de ingresos de un mismo paciente en diferentes centros o de ingresos sucesivos sin que se llegara a realizar el tratamiento del aneurisma podría hacer disminuir el número de enfermos tratados, y que también otros factores permitirían explicar estas diferencias con nuestro país, tales como la ausencia de presión económica para llevar o no a cabo un tratamiento en los centros que han participado en este registro, dado que todos pertenecen a la red sanitaria pública nacional, o quizás a la diferente situación en cuanto a la presión de reclamaciones que puede existir en nuestro país, y que hace que en Estados Unidos se pueda practicar una medicina más defensiva. En cuanto al tipo de tratamiento cabe destacar la mayor frecuencia del tratamiento endovascular en nuestro país, tendencia que se repite en Europa, en comparación con los estudios americanos, donde el tratamiento endovascular se reserva a aquellos casos con peor pronóstico. Por otro lado, la estancia media (mediana de 23 días para enfermos tratados) es muy similar a la reportada para enfermos con HSA en el 2003 en Estados Unidos, siendo ésta de 20 días para los tratados quirúrgicamente y 17 para los tratados endovascularmente ${ }^{32}$. Dada la baja disponibi- lidad de centros de derivación, fundamentalmente de centros de rehabilitación, en nuestro medio, este dato es muy significativo e incluso puede causar perplejidad, y denota, por otro lado, el esfuerzo realizado por los médicos de los centros que han participado en este estudio.

En cuanto a los resultados globales comparados con otras series españolas previamente publicadas ${ }^{14,18}$, no se observa una variación en el resultado final, siendo muy similares las tasas de morbilidad y mortalidad, a pesar de que el porcentaje de enfermos tratados endovascularmente haya aumentado considerablemente, y la precocidad del tratamiento haya sido mayor (64\% tratado en los primeros 3 días tras el sangrado). Aunque no se pueden extraer conclusiones acerca de si un tratamiento es mejor o peor que el otro, ya que el estudio no fue diseñado para ello, parece evidente que el tipo de tratamiento aplicado a la causa del sangrado no produce un efecto importante sobre el resultado final de la enfermedad, siempre que se realice siguiendo un estándar de calidad adecuado ${ }^{12}$. Por ello, en nuestra opinión, sería importante prescindir de debates espúreos sobre la mayor conveniencia de uno $\mathrm{u}$ otro tratamiento, y dirigir la investigación a aliviar el daño producido por la propia hemorragia o sus complicaciones, ya que en la gravedad del sangrado inicial reside el principal factor pronóstico de esta enfermedad ${ }^{13,14}$.

Otro dato a tener en cuenta es la variación en cuanto al porcentaje que representa la HSAi en esta serie, más próxima al $15 \%$ descrito internacionalmente, que a cifras consignadas previamente en nuestro medio ${ }^{8,15,18}$. La mejoría en los métodos diagnósticos, tanto en la mayor resolución de la angiografía, como del angio-TC y la angio-RM, han hecho probablemente que este porcentaje disminuya en nuestro país, a pesar de que sigue siendo discretamente más alto que en otras zonas del mundo.

A pesar de que este registro representa una oportunidad inmejorable a la hora de estudiar un número importante de características de esta enfermedad, existen también limitaciones a señalar. La principal de ellas es que no se trata de un registro implantado universalmente en nuestro país. Los datos aquí presentados reflejan la situación de los centros que han participado y aunque son muchos, no son probablemente extrapolables a todos los hospitales españoles. Por otro lado, estos datos no se han auditado externamente, hecho que puede restar validez a las conclusiones que se obtengan del registro. Sin embargo, la mayor parte de las series de la literatura tampoco han sido sometidas a auditoría. La ausencia de conflictos de interés o de financiación externa de la inclusión de enfermos en la base hace que los datos sean tan fiables como los de cualquier otra serie de enfermos anteriormente publicada.

En resumen, presentamos los resultados de tres años de trabajo en una base de datos multicéntrica, hospitalaria de enfermos no seleccionados con HSA. Queremos con ello 
llamar la atención de la comunidad neuroquirúrgica sobre esta enfermedad y despertar el interés por conseguir la realización de registros a nivel nacional de éste y otros tipos de patología. Demostramos que esta empresa es factible y que su consecución daría importante información sobre la frecuencia, complejidad, tipo de tratamiento empleado y resultados en esta enfermedad. El Grupo de Patología Vascular intentará con futuros trabajos explicar de forma más detallada aspectos relacionados con el estudio de esta base y de la HSA que se han descrito de manera preliminar en este trabajo. El mantenimiento del compromiso de los diferentes investigadores que han participado en este trabajo cooperativo, así como la inclusión de nuevos centros, será clave para que esta base de datos se confirme de forma definitiva en un registro hospitalario de esta enfermedad.

\section{Agradecimientos}

A Ramiro Díez Lobato por revisar e inspirar este manuscrito.

A la SENEC por ayudar a la elaboración y mantenimiento del registro.

Al Grupo de Patología Vascular de la SENEC y en particular a Jordi Vilalta, Marta Brell y $\mathrm{M}^{\mathrm{a}} \mathrm{Fe}$ Muñoz por colaborar activamente en el mantenimiento del registro.

Este proyecto ha sido además en parte financiado por ayudas de investigación en curso en algunos centros tales como: Fundación Mutua Madrileña 2007/082, Fondo de Investigaciones Sanitarias FIS PI 070152, Hospital 12 de Octubre.

\section{Bibliografía}

1. Avidan, A., Weissman, C., Sprung, C.L.: An internet web site as a data collection platform for multicenter research. Anesth Analg 2005; 100: 506-511.

2. Broderick, J.P., Brott, T.G., Duldner, J.E., Tomsick, T., Leach, A.: Initial and recurrent bleeding are the major causes of death following subarachnoid hemorrhage. Stroke 1994; 25: $1342-1347$.

3. Cooper, C.J., Cooper, S.P., del Junco, D.J., Shipp, E.M., Whitworth, R., Cooper, S.R.: Web-based data collection: detailed methods of a questionnaire and data gathering tool. Epidemiol Perspect Innov 2006; 3: 1-

4. Cross, D.T., III, Tirschwell, D.L., Clark, M.A., et al.: Mortality rates after subarachnoid hemorrhage: variations according to hospital case volume in 18 states. J Neurosurg 2003; 99: 810-817.

5. Deruty, R., Pelissou-Guyotat, I., Mottolese, C., Amat, D., Bognar, L.: Level of consciousness and age as prognostic factors in aneurysmal SAH. Acta Neurochir (Wien) 1995; 132: $1-8$.

6). Fisher, C.M., Kistler, J.P., Davis, J.M.: Relation of cerebral vasospasm to subarachnoid hemorrhage visualized by computerized tomographic scanning. Neurosurgery 1980; 6: $1-9$.

7. Fogelholm, R., Hernesniemi, J., Vapalahti, M.: Impact of early surgery on outcome after aneurysmal subarachnoid hemorrhage. A population-based study. Stroke 1993; 24: 1649-1654.

8. Gómez, P.A., Lobato, R.D., Rivas, J.J., et al.: Subarachnoid haemorrhage of unknown aetiology. Acta Neurochir (Wien) 1989; 101: 35-41.

9. Hoh, B.L., Curry, W.T., Jr., Carter, B.S., Ogilvy, C.S.: Computed tomographic demonstrated infarcts after surgical and endovascular treatment of aneurysmal subarachnoid hemorrhage. Acta Neurochir (Wien) 2004; 146: 1177-1183.

10. Inagawa, T., Shibukawa, M., Inokuchi, F., Tokuda, Y., Okada, Y., Okada, K.: Primary intracerebral and aneurysmal subarachnoid hemorrhage in Izumo City, Japan. Part II: management and surgical outcome. J Neurosurg 2000; 93 : 967-975.

11. Jennett, B., Bond, M.: Assessment of outcome after severe brain damage. Lancet 1975; 1: 480-484.

12. Koivisto, T., Vanninen, R., Hurskainen, H., Saari, T., Hernesniemi, J., Vapalahti, M.: Outcomes of early endovascular versus surgical treatment of ruptured cerebral aneurysms. A prospective randomized study. Stroke 2000; 31: 2369-2377.

13. Lagares, A., Gómez, P.A., Alén, J.F., Lobato, R.D., Campollo, J.: Global cerebral edema after subarachnoid hemorrhage. Stroke 2002; 33: 2153-2154.

14. Lagares, A., Gómez, P.A., Lobato, R.D., Alén, J.F., Alday, R., Campollo, J.: Prognostic factors on hospital admission after spontaneous subarachnoid haemorrhage. Acta Neurochir (Wien) 2001; 143: 665-672.

15. Lagares, A., Gómez, P.A., Lobato, R.D.et al.: [Idiopathic subarachnoid hemorrhage; comparison of different bleeding patterns and long-term outcome]. Neurocirugía 2002; 13: 110-119.

16. Lallas, C.D., Preminger, G.M., Pearle, M.S., et al.: Internet based multi-institutional clinical research: a convenient and secure option. J Urol 2004; 171: 1880-1885.

17. Marks, R., Bristol, H., Conlon, M., Pepine, C.J.: Enhancing clinical trials on the internet: lessons from INVEST. Clin Cardiol 2001; 24: V17-V23.

18. Martínez-Mañas, R., Ibánez, G., Macho, J., Gaston, F., Ferrer, E.: [A study of 234 patients with subarachnoid hemorrhage of aneurysmic and cryptogenic origin]. Neurocirugía 2002; 13: 181-193.

19. Molyneux, A., Kerr, R., Stratton, I., et al.: International Subarachnoid Aneurysm Trial (ISAT) of neurosurgical clipping versus endovascular coiling in 2143 patients with ruptured intracranial aneurysms: a randomized trial. J Stroke Cerebrovasc Dis 2002; 11: 304-314.

20. Molyneux, A.J., Kerr, R.S., Yu, L.M., et al.: Interna- 
tional subarachnoid aneurysm trial (ISAT) of neurosurgical clipping versus endovascular coiling in 2143 patients with ruptured intracranial aneurysms: a randomised comparison of effects on survival, dependency, seizures, rebleeding, subgroups, and aneurysm occlusion. Lancet 2005; 366: 809-817.

21. Muñoz-Sánchez, M.A., García-Alfaro, C., MuñozLópez, A., et al.: [The EHSA project: the study of spontaneous subarachnoid haemorrhages in Andalusia. Incidence and results]. Rev Neurol 2003; 36: 301-306.

22. Nilsson, O.G., Lindgren, A., Stahl, N., Brandt, L., Saveland, H.: Incidence of intracerebral and subarachnoid haemorrhage in southern Sweden. J Neurol Neurosurg Psychiatry 2000; 69: 601-607.

23. Ogilvy, C.S., Carter, B.S.: A proposed comprehensive grading system to predict outcome for surgical management of intracranial aneurysms. Neurosurgery 1998; 42: 959-968.

24. Ogungbo, B., Gregson, B.A., Blackburn, A., Mendelow, A.D.: Trends over time in the management of subarachnoid haemorrhage in newcastle: review of 1609 patients. Br J Neurosurg 2001; 15: 388-395.

25. Paul, J., Seib, R., Prescott, T.: The Internet and clinical trials: background, online resources, examples and issues. $\mathrm{J}$ Med Internet Res 2005; 7: e5-

26. Qureshi, A.I., Suri, M.F., Nasar, A.et al.: Trends in hospitalization and mortality for subarachnoid hemorrhage and unruptured aneurysms in the United States. Neurosurgery 2005; 57: 1-8.

27. Rinkel, G.J., Wijdicks, E.F., Vermeulen, M., Hasan, D., Brouwers, P.J., van Gijn, J.: The clinical course of perimesencephalic nonaneurysmal subarachnoid hemorrhage. Ann Neurol 1991; 29: 463-468.

28. Rinkel, G.J., Wijdicks, E.F., Vermeulen, M.et al.: Nonaneurysmal perimesencephalic subarachnoid hemorrhage: CT and MR patterns that differ from aneurysmal rupture. AJNR Am J Neuroradiol 1991; 12: 829-834.

29. Rosengart, A.J., Schultheiss, K.E., Tolentino, J., Macdonald, R.L.: Prognostic factors for outcome in patients with aneurysmal subarachnoid hemorrhage. Stroke 2007; 38: 23152321.

30. Rothwell, P.M., Coull, A.J., Giles, M.F., et al.: Change in stroke incidence, mortality, case-fatality, severity, and risk factors in Oxfordshire, UK from 1981 to 2004 (Oxford Vascular Study). Lancet 2004; 363: 1925-1933.
31. Saveland, H., Brandt, L.: Which are the major determinants for outcome in aneurysmal subarachnoid hemorrhage? A prospective total management study from a strictly unselected series. Acta Neurol Scand 1994; 90: 245-250.

32. Shea, A.M., Reed, S.D., Curtis, L.H., Alexander, M.J., Villani, J.J., Schulman, K.A.: Characteristics of nontraumatic subarachnoid hemorrhage in the United States in 2003. Neurosurgery 2007; 61: 1131-1137.

33. Teasdale, G., Jennett, B.: Assessment of coma and impaired consciousness. A practical scale. Lancet 1974; 2: 8184.

34. Teasdale, G.M., Drake, C.G., Hunt, W., et al.: A universal subarachnoid hemorrhage scale: report of a committee of the World Federation of Neurosurgical Societies. J Neurol Neurosurg Psychiatry 1988; 51: 1457-

35. Topcuoglu, M.A., Ogilvy, C.S., Carter, B.S., Buonanno, F.S., Koroshetz, W.J., Singhal, A.B.: Subarachnoid hemorrhage without evident cause on initial angiography studies: diagnostic yield of subsequent angiography and other neuroimaging tests. J Neurosurg 2003; 98: 1235-1240.

36. van Gijn, J., Kerr, R.S., Rinkel, G.J.: Subarachnoid haemorrhage. Lancet 2007; 369: 306-318.

37. Vilalta, J.: [Prognosis of patients treated with the clipping of brain aneurysm following subarachnoid hemorrhage]. Med Clin (Barc ) 1999; 113: 478-

38. Wolfe, C.D., Rudd, A.G., Howard, R.et al.: Incidence and case fatality rates of stroke subtypes in a multiethnic population: the South London Stroke Register. J Neurol Neurosurg Psychiatry 2002; 72: 211-216.

Lagares, A.; de Toledo, P.; Fernández-Alén, J.A.; Ibáñez, J.; Arikan, F.; Sarabia, R,; Ballenilla, F.; Gabarros, A.; Horcajadas, A.; Rodríguez-Boto, G.; de la Lama, A.; Maillo, A.; Delgado, P.; Llacer, J.L.; Domínguez, J.; Arrese, I.: Base de datos multicéntrica de hemorragia subaracnoidea espontánea del Grupo de Trabajo de Patología Vascular de la Sociedad Española de Neurocirugía: presentación, criterios de inclusión y desarrollo de una base de datos en internet. Neurocirugía 2008; 19: 405-415.

Correspondencia postal: Alfonso Lagares, Servicio de Neurocirugía, Hospital 12 de Octubre, Avda. de Cordoba s/n. 28041 Madrid. 